\title{
Evaluation of Online Health and Wellness Resources for Healthcare Professionals
}

\author{
doi:10.3991/ijac.v4i2.1659 \\ C.J. MacDonald ${ }^{1}$, D. Archibald ${ }^{1}$ H. Kellam ${ }^{1}$, R. Sun ${ }^{1}$, E. Stodel ${ }^{2}$ and D. Puddester ${ }^{1}$ \\ ${ }^{1}$ University of Ottawa, Ottawa, Canada \\ ${ }^{2}$ Learning 4 Excellence, Ottawa, Canada
}

\begin{abstract}
Two online resources, ePhysicianHealth.com and eWorkplaceHealth.com, were developed to help physicians and other healthcare professionals improve their health and well-being by providing them with relevant, up-to-date support and resources at no cost and with anonymous access. ePhysicianHealth.com is the world's first comprehensive, online physician health and wellness resource designed to help physicians and medical students be resilient in their professional and personal lives. eWorkplaceHealth.com is a new and original resource aimed to increase awareness and understanding of the issues and factors that may affect healthcare professionals' health at work. ePhysicianHealth.com includes 14 modules in French and English and eWorkplaceHealth.com comprises a four-module program in English. A program evaluation using quantitative and qualitative methods was conducted to obtain evidence regarding the users' perceptions of the learning resources and their impact on the specified outcomes.
\end{abstract}

Most participants felt they gained new knowledge from ePhysicianHealth.com and eWorkplaceHealth.com. They felt they had access to useful information through the resources, which increased their awareness of the various issues facing physicians today and where they can go to get assistance when in need.

Index Terms-physician health and wellness, workplace health, eLearning, resilience, medical education, healthcare professionals

\section{INTRODUCTION}

The quality and sustainability of the healthcare system in Canada is primarily dependent on the healthcare professionals who provide care. If the system is to remain strong, it is critical that providers are resilient and healthy. Demands on the Canadian healthcare system continue to escalate and this has led to extremely heavy workloads for those who work within it and high levels of burnout. One study found that $46 \%$ of Canadian physicians are in advanced stages of burnout [1]. Another revealed that many new nurses are leaving their jobs within two years of graduation and have symptoms of burnout, depression, and emotional exhaustion [2]. As well, Dyrbye et al. [3] found an association between burnout and an increased likelihood of suicidal ideation in medical students. Not only are there concerns for the healthcare professionals themselves but their health can also impact patient care and satisfaction [4]. Further, the average number of days lost due to illness or disability is about double for those who work in healthcare than in any other occupation in Canada [5]. The importance of improving health and wellbeing of healthcare professionals and developing resil- iency cannot be overstated. In this context, ePhysicianHealth.com and eWorkplaceHealth.com were developed to provide relevant, up-to-date support and resources on topics relevant to the health and well-being of physicians and other healthcare professionals.

ePhysicianHealth.com includes 14 modules in French and English on topics such as weight, nutrition, and fitness; anxiety; substance use disorders; and disruptive behaviour. eWorkplaceHealth.com comprises a four-module program designed to increase practical awareness and understanding of the factors that impact health at work. Since this evaluation, additional modules have been added to eWorkplaceHealth.com to help teams work together more effectively.

The purpose of this paper is to report on the evaluation of these two resources. The following research questions guided the evaluation: (1) What were the participants' experiences using the two learning resources? (2) Did the participants acquire new knowledge and skills as a result of using the learning resources? (3) Did the participants apply anything they learned to improve their own health and well-being or that of other healthcare professionals?

\section{METHODOLOGY}

\section{A. The Framework}

$W(e) L e a r n$ [6], a framework created to guide the design, development, delivery, and evaluation of interprofessional education, was adopted as the evaluation framework in this study. W(e)Learn (refer to Fig. 1) proposes four critical dimensions of an effective programstructure, content, media, and service-required to elicit four levels of outcome, the pinnacle of which is organizational change and the resulting improvement in care delivery that promotes patient well-being.

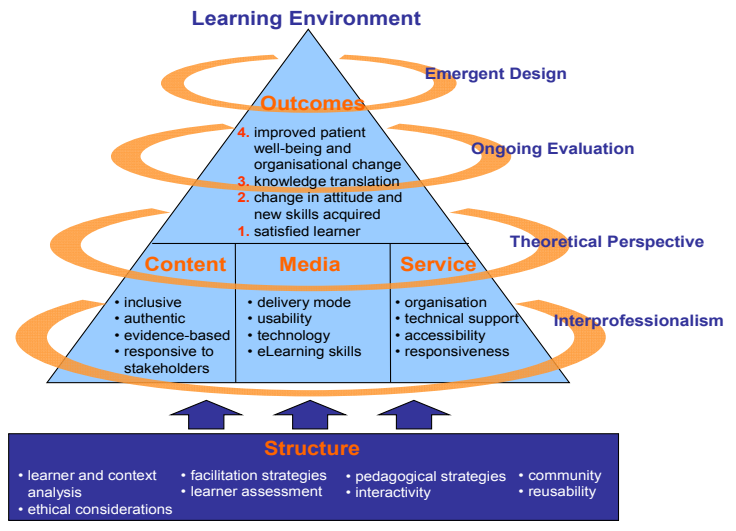

Figure 1. W(e)Learn 


\section{B. Procedures}

Following an invitation to participate in this study, 45 healthcare professionals agreed to evaluate the resources and complete a demographic survey. Of these, $16(36 \%)$ were medical students, $13(29 \%)$ were residents, $11(25 \%)$ were practicing physicians, $2(4 \%)$ were nurses, $2(4 \%)$ were physiotherapists, and $1(2 \%)$ was a medical

educator. There were $31(69 \%)$ female participants and $14(31 \%)$ male participants.

Participants were asked to evaluate at least two modules by completing the module and then filling in an online post-module survey-the W(e)Learn evaluation tool. Thirty of the 45 participants $(67 \%)$ evaluated two modules and a further three participants $(7 \%)$ evaluated a single module providing 63 reviews of modules (refer to Table 1). Follow-up interviews (seven focus groups and two individual interviews) were conducted with 18 of the 33 (55\%) participants to elicit richer information to help answer the research questions. This is an example of a concurrent triangulation mixed methods research design [7]. We used this design to compare and contrast the quantitative results of the online surveys with the qualitative findings of the interviews. By using qualitative data to supplement, complement, corroborate and/or contradict the quantitative survey data, a better understanding of the participants' learning experience can be attained.

\section{Data Collection}

At the start of the research, a Demographic Survey was administered to gather information regarding participants' computer skills and experience and their attitudes towards online learning. Then, after the completion of a module, participants were asked to complete the W(e)Learn evaluation tool. The W(e)Learn evaluation tool is designed to collect data related to the W(e)Learn constructs

TABLE I.

NUMBER OF REVIEWS FOR EACH MODULE

\begin{tabular}{|l|c|c|}
\hline \multirow{2}{*}{ Modules } & \multicolumn{2}{|c|}{$\begin{array}{c}\text { Percentage/number } \\
\text { of reviews }\end{array}$} \\
\cline { 2 - 3 } & $\mathbf{\%}$ & $\#$ \\
\hline substance use disorders & 6.3 & 4 \\
\hline weight, nutrition, and fitness & 15.9 & 10 \\
\hline depression, burnout, and suicide & 7.9 & 5 \\
\hline Anxiety & 12.7 & 8 \\
\hline Resilience & 17.5 & 11 \\
\hline relationship with self & 9.5 & 6 \\
\hline Boundaries & 6.3 & 4 \\
\hline primary care & 1.6 & 1 \\
\hline disruptive behaviour: physician leaders & 1.6 & 1 \\
\hline disruptive behaviour: medical students & 3.2 & 2 \\
\hline disruptive behaviour: residents & 4.8 & 3 \\
\hline disruptive behaviour: practising physicians & 0.0 & 0 \\
\hline disruptive behaviour: interprofessional teams & 4.8 & 3 \\
\hline Total for ePhysicianHealth.com & $\mathbf{9 2}$ & $\mathbf{5 8}$ \\
\hline Introduction & 1.6 & 1 \\
\hline taking care of yourself & 3.2 & 2 \\
\hline taking care of others & 1.6 & 1 \\
\hline taking care of the work & 1.6 & 1 \\
\hline Total for eWorkplaceHealth.com & $\mathbf{8}$ & $\mathbf{5}$ \\
\hline Total for Both Resources & $\mathbf{1 0 0}$ & $\mathbf{6 3}$ \\
\hline & & \\
\hline
\end{tabular}

of content, media, service, structure, and outcomes. The surveys were delivered and completed online using Survey Monkey ${ }^{\mathrm{TM}}$.

All participants who completed two modules and the affiliated post-module evaluation were invited to take part in a follow-up interview. Everyone who accepted the invitation was interviewed if schedules permitted. Follow-up focus group and individual interviews were conducted with just over half the participants $(\mathrm{N}=18)$ in order to gain a deeper understanding of their experiences completing the modules. All interview participants were medical students and residents. Interviews were conducted face-toface or by telephone depending on the participant's preference. Interview questions were based on the findings from the surveys. Each interview lasted approximately 25-35 minutes, was audio-taped, and transcribed verbatim.

\section{Data Analysis}

Quantitative data analysis involved calculating frequency and descriptive statistics for the participants' responses on the closed-answer survey items of the demographic questionnaire and the W(e)Learn evaluation tool.

Qualitative data from the interviews were initially managed by QSR NVivo 8 software to identify themes. Two researchers independently analyzed the data and then compared findings. The categories, themes, and patterns that emerged in this process were evaluated for their credibility. At the same time, the researchers searched through the data for disconfirming instances and alternative explanations.

\section{FINDINGS}

The findings of the evaluation are presented under the $W(e)$ Learn constructs of content, media, service, structure, and outcomes. Frequency data from the surveys are presented and illustrated with quotations from the interviews. The findings under each construct have been organized by theme using qualitative thematic analysis.

\section{A. Content}

Four sub-themes emerged concerning the content of the learning resources: Relevant, Learning Objectives, Depth, and Subject Matter Experts.

The majority (94\%) of the participants interviewed reported that the content was relevant, authentic, and applicable to their personal and professional lives. One participant reflected:

I do believe it [the content] is authentic because I already know of some colleagues that are having some problems with substances. In regards to the disruptive behaviour of a resident, I think it is both relevant and authentic because I have already had to deal with people that I know are going to be disruptive residents. Now in regards to resolving conflict with them, I will be more appropriate and successful. (medical student 1)

Participants could personally relate to the content, which increased the impact of the modules and engaged them in the learning process. One resident indicated the information is relevant for all members of the healthcare team. Another, who is an expert in diet and exercise, indicated that the exercise, nutrition, and fitness module, while not beneficial for him, would be an excellent tool for some of his colleagues. However, another two partici- 
PAPER

Evaluation of Online HeAlth AND Wellness Resources for HeAlthCARE Professionals

pants said that while they found the modules engaging, more concrete and applicable information would make them more relevant. For example, one participant wanted quick healthy recipes and ideas on how to change things if you are already someone who lives a healthy life but are a busy working mother. Several participants identified the videos as important tools for presenting content in an authentic manner.

The majority of participants agreed the learning objectives stated at the beginning of each module were met. A few admitted they could not recall what the learning objectives were. However, one resident (2) was concerned that the depth of the content did not meet the learning objectives and another indicated that the degree to which the learning objectives were met varied across modules.

I am still not really clear. For the Disruptive Behaviour [module], it depends who you are to know the strategy and how to deal with it.... whether you are the med student or the resident or whatever. I still didn't get a clear picture by the end of it. The depression one was fantastic though, that one gave you clear direction.

In $95 \%$ of the surveys, the participants agreed that the content of the modules is of appropriate length and depth. As well, eight of the participants interviewed reported the modules were an appropriate length and provided suitable content depth. One medical student (6) stated, "I found the length of the modules perfect. Just long enough that you could get all the information that you really wanted to get out of it but it was not too long that your attention started to wane".

Conversely, several participants questioned the depth of the content. Some felt the longer modules were overwhelming and could be divided into separate modules. One medical student (6) commented, "I think I was pleased with the depth of the [Weight, Nutrition, and Fitness module]. The Depression [module] was ... an interesting topic and I think it lacked a little bit of depth".

In $98 \%$ of the surveys, the participants agreed that the subject matter experts who developed the content were qualified and experienced. One resident (6) mentioned that she appreciated the variety of experts used:

I enjoyed the fact that experts came from various backgrounds. To one extent I remember in the resilience section a family medicine practitioner in Western Ontario talking about her job as a mentor and a program director in helping her colleagues and trainees. Then you had another expert who has a psychiatry background.

\section{B. Media}

Several sub-themes emerged relating to media: Realism, Personal Stories, Professional Application, Use of Videos, and Exercises.

Participants repeatedly indicated the videos added realism to the content by presenting information in a new and different fashion: "Overall, the videos ... are fantastic. They make it easy to follow and really illustrate what is going on. Especially [in] the Disruptive Behaviour [modules]. It really demonstrates what is going on" (resident 2). In $81 \%$ of the surveys, participants indicated that the videos promoted a more meaningful learning experience. A resident said the videos opened her eyes and allowed her to see new perspectives. Another said the videos were rich with examples that helped him focus on the information that was being presented. One resident alleged that while he believed the content of some of the scripted stories was excellent, the delivery and acting in the videos hurt their credibility.

The majority of participants said the videos involving personal stories allowed them to better identify with the information: "it's very sobering and adds a lot of credibility and improves acceptance of the material". In $90 \%$ of the surveys, participants said the videos promoted selfawareness. One resident asserted that the videos of personal stories were shocking and brought the content to life.

In $86 \%$ of the surveys, participants said the videos presented knowledge they could apply in their professional lives. A resident (5) mentioned: "I recall the one [module] on Boundaries and it gave some good examples in the videos that I found quite helpful in thinking about how you define boundaries".

Although the videos on disruptive behaviour made some participants more aware, due to the hierarchy in medicine many reported they still would not feel comfortable confronting their "superiors" if they witnessed disruptive behaviour.

Participants did not like the introductory videos that were "talking heads". In general, participants felt video should only be used when the content could not be presented in written form. If the video was just someone conveying information that could be read faster and more efficiently, participants did not see added value and felt the videos wasted time. More generally, a number of participants felt the videos were too long.

Several participants listed the exercises as their favourite part of the modules since they allowed them to test their understanding and assimilate the information. A resident (6) mentioned his favourite part of the modules he reviewed was in the Resilience module: "the five-minute meditation exercise with square breathing. I thought that was insightful and fun to do it in real-time. I enjoyed that and learned from it".

Others said they did not enjoy the exercises, found them too long, and skipped over them. One participant recommended making the exercises shorter to motivate participation.

\section{Service}

One sub-theme emerged relating to service: Resources. In $97 \%$ of the surveys, participants agreed that the modules included sufficient online resources. Participants said the links added value, variety, and were a fun way to reinforce information. Participants were unanimous in their praise for the resources that were found throughout the modules of ePhysicianHealth.com and eWorkplaceHealth.com. The resources included websites, books, articles, workshops, and support tools. Participants said the links to external websites added value and variety, as well as being a fun way to reinforce information in the learning resource. One resident (7) explained, "They [website links] were easy to access and the ones that I took a look at were useful ... They were not redundant but added a nice link if you were looking for more information".

Several participants mentioned the links provided a different viewpoint on the topic and some included interactive activities. For example, one resident (6) testified:

Those websites were of various backgrounds and addressed the issues with a different spin, which was inter- 
PAPER

esting. I remember this two page questionnaire .... where you were scored on different habits on your own mental health relating to resilience. At the end of the module you get this PDF document generated. I kept both those documents on my hard drive for reference.

Resident (7) visited the same website and added: "I also really liked that there was something being generated as you went along that you could save as a document for later".

\section{Structure}

Three sub-themes related to structure emerged: Navigation, Flexibility, and Engaging.

Participants' responses regarding navigation were inconsistent and, at times, contradictory. In the post-module survey, $92 \%$ of the responses indicated that the participants found the resource easy to navigate. For example, a medical student (5) recounted, "The layout was really good, visually appealing and easy to go through the different paths". Similarly, a resident (8) described the ease of navigating ePhysicianHealth.com:

Overall, I thought it was really well done. The presentation was very good and easy to navigate. I found the way that you progressed through the modules to be very user friendly as well so you could complete sections at a time. It was very straightforward, relaxing, and easy to get through.

Conversely, nine of eighteen interview participants indicated that the modules were at times difficult and/or confusing to navigate. A main problem was they were often confused with the way the tabs and links were organized on the page. One resident (11) described this:

I could click on fifteen things at once so at the end even though I went through it I wasn't sure if I had touched on everything. There seemed to be a lot of layers. So that was the only thing. Did I get all the information that I could? Did I miss three of the fifteen things that you could put your mouse over and click on?

One resident suggested adding a graphical index to highlight the module content on one page. Similarly, participants suggested the modules were so rich with content that they would benefit from a more linear design.

Participants appreciated the flexibility of ePhysicianHealth.com and eWorkplaceHealth.com; they could work on the modules at any time and place convenient to them. They also valued the fact they could work at their own pace. Several liked that they could choose to access links that directed them to extra resources or choose to skip over them. One appreciated that he could work on his own time rather than being forced to progress through the modules in a predetermined way.

In $70 \%$ of the surveys, participants indicated the module kept their interest; in $20 \%$ of the surveys, participants indicated this was not the case. Participants certainly preferred some modules over others and found some more engaging than others. The clinical vignettes and personal storytelling in the videos, the exercises, and using and applying the new knowledge in interactive activities were elements participants indicated helped keep their interest.

The Anxiety [module] held my attention. ... Those clinical vignettes of people telling their story were interesting and a nice application to tie things together. The Nutrition module felt not quite as logical in terms of its ...information being presented but without a solid way of applying it or personal stories or something to keep my interest. I felt it dragged... (resident 9)

Another resident (6) explained: "I would say yes [the modules held my interest]. The Resilience module more so than the one on Relationships, which was interesting but I thought the one on Relationships covered the essentials of its matter early on into the module".

Another resident (11) discussed her interest level with regard to the modules:

At one point during the Resilience module I got part way through it and found there was still more but then I remembered that we were going through an acronym. Also in the Depression one I thought was a good split in the three areas and it shifted quickly enough that it kept my attention.

\section{E. Outcomes}

The outcomes of the evaluation are presented in response to the research questions.

What were the participants' experiences using the two learning resources? Participants unanimously agreed that the learning resources provided a novel, convenient, easy, and enjoyable way to learn. Many had fun using the resources, valued the expertise of the subject matter experts involved, and thought the content was relevant and authentic. Participants found the modules were interactive. In $62 \%$ of the surveys, participants indicated that the resources included relevant and appropriate use of technology. They were enthusiastic about the variety of teaching tools and strategies employed and gave examples of how the videos, exercises, and resources were helpful. One resident indicated the best thing about the resources was that they provided accessible and free quality content. In $58 \%$ of the surveys, participants agreed that the choice of technology tools allowed them to learn using their preferred learning style.

Did the participants acquire new knowledge and skills as a result of using the learning resources? Ten of the eighteen interview participants said they acquired new knowledge as a result of doing the module(s). In $83 \%$ of the surveys, participants agreed that they now understood new principles. Several participants mentioned that the real power of the learning resources was that they reinforced previous knowledge. They felt this review would help them apply the knowledge in the appropriate context in the future.

Did the participants apply anything they learned to improve their own health and well-being or that of other healthcare professionals? If so, what? While many indicated they had not yet applied their new knowledge, many indicated they intend to share the resources with colleagues and apply what they have learned in the future. Primarily, participants indicated that their new knowledge related to an increased awareness of the various issues facing physicians today and the avenues available to them for assistance. The participants provided some examples of how they had applied what they had learned to date. One resident became more aware of how to make herself more resilient. Another found practical tips on how to maintain relationships. Another indicated she was now taking better care of herself and making more of an effort to set priorities in her life. Others indicated they were going to apply what they had learned when working with 
PAPER

patients. Several participants said that they would definitely revisit the learning resources in the future.

In summary, overall, participants agreed that reviewing the resources provided them with information and tools that benefited them both personally and professionally. They were unanimous that the modules they examined provided relevant information and an excellent foundation for further study. Furthermore, participants found that the modules were presented in an interactive manner and were a convenient and easy way to learn. Participants were enthusiastic with regard to the variety of teaching tools and strategies the modules employed. They particularly appreciated the videos and noted the learning value of the realistic scenarios the videos portrayed. In addition to the positive feedback, there were also a few inconsistencies in the participants' reactions to the modules, as well as several recommendations for how the learning resources can be improved.

\section{CONCLUSIONS AND DISCUSSION}

Most participants felt they gained new knowledge from their involvement with ePhysicianHealth.com and eWorkplaceHealth.com. In many cases, this knowledge came in the form of useful information or increased awareness but stopped short of acquiring new strategies to deal with the issue or situation. Primarily, participants indicated that the learning resources mainly increased their awareness of the various issues facing physicians today, and the fact that there are avenues where they can go to get assistance. Several participants suggested the real power of ePhysicianHealth.com and eWorkplaceHealth.com are that they reinforced knowledge and skills they already had. They felt this review would help them apply the knowledge in the appropriate context in the future. Moreover, participants appreciated that although the content was not new, having it summarized and organized specifically for physicians and medical students was a new and useful spin on old material.

Many participants said they enjoyed the various media used in the learning resources. They expressed how they appreciated the fact that not only were there readings but interactive exercises, case studies, scenarios, quizzes, and the opportunity to watch videos.

The videos explaining how to choose a running shoe, how to read food labels, and the one on suicide were mentioned several times as being useful. The main message regarding the use of video in these resources was that they were effective but only when the message could not be delivered faster and easier in text. In essence, the participants were advocating the use of stories and personal testimonies as opposed to 'talking heads' delivering content. They particularly liked personal stories that reinforced the educational power of the videos but emphasized they were only effective if they were realistic. As well, several participants felt many of the videos were too long and a better job could be done to edit them to a more appropriate length.

There were mixed messages on how easy the resources are to navigate. Several participants said it was well organized and easy to navigate, while others reported they found the modules confusing, were frustrated not knowing where to go next, and found too much content on one page. Six out of the eighteen participants identified that the navigation was their least favourite part of ePhysi-
cianHealth.com. A few participants suggested that a simple reorganization of the site might provide them with a better idea of where to navigate and locate information. They recommended strategies such as a more comprehensive table of contents and/or a graphic map that would allow them to see everything at a glance. Other recommendations included a more linear design and all the links in one place.

Although most participants reported that either they had not yet transferred their learning to the workplace or when they had it was limited to sharing the resource with a colleague, many reported they thought they would use the information learned from the resource in their future career. Moreover, many of the examples participants provided explaining what they thought they would use, and how, indicated that transferring their learning from ePhysicianHealth.com to their work situations had the potential to either benefit the patient and/or improve the workplace situation and healthcare organization. As the participants interviewed were medical students and residents, following up with these participants when they are practicing physicians would provide a better indication on whether or not they actually transferred any of the knowledge or skills from the learning resource into practice.

\section{RECOMMENDATIONS FOR IMPROVEMENT}

Participants provided the following recommendations for improving the resources:

- Include a graphical content map for each module to aid navigation.

- Move the navigation tabs to the side rather than across the top. Make them more visible.

- Only use video to convey information that cannot be delivered as effectively using text.

- Use video to share engaging stories and personal testimonies as opposed to 'talking heads'.

- Edit videos so they last 30-60 seconds.

- Keep interactive exercises short and to the point.

- In addition to providing information to increase awareness of topics, include more skills and practical ideas on how to manage or treat symptoms or situations.

- Reduce content on some of the pages.

- Market the resource so that the appropriate audience realizes its availability.

\section{SUMMARY}

Both ePhysicianHealth.com and eWorkplaceHealth.com are useful resources for healthcare professionals and trainees related to health and well-being. The resources are designed to provide anonymous access to support and resources for individuals in need, as well as those looking to improve their health and well-being. Many of the participants involved in this evaluation did not have specific healthcare needs. It would be valuable to obtain feedback on the modules from individuals who access these resources on their own accord to meet their own specific needs. Moreover, more insights would be obtained from longer term follow up with the participants and/or users regarding the application of knowledge gained from these resources in their work environment and/or personal lives. 
PAPER

Evaluation of Online HeAlth AND Wellness Resources for HeAlthCARE Professionals

ePhysicianHealth.com was the 2010 International ELearning awards winner for the Business/Professional ELearning category.

\section{REFERENCES}

[1] Canadian Medical Association, 2003. CMA Study on Physician Burnout. Retrieved July 14, 2009 from http://www.cma.ca/ multimedia/staticContent/HTML/N0/12/PhysicianHealth/news/ pdf/Burn-out.pdf

[2] Cho, J., Laschinger, H.K.S., \& Wong, C., 2006. Workplace Empowerment, Work Engagement and Organizational Commitment of New Graduate Nurses. Canadian Journal of Nursing Leadership, 19(3), pp. 43-60.

[3] Dyrbye, L.N., et al., 2008. Burnout and Suicidal Ideation Among U.S. Medical Students. Annals of Internal Medicine, 149(5), pp. 334-341.

[4] Halbesleben, J., \& Rathert, C., 2008. Linking Physician Burnout and Patient Outcomes: Exploring the Dyadic Relationship Between Physicians and Patients. Health Care Management Review, 33(1), pp. 29-39.

[5] Statistics Canada, 2008. Work Absence Rates. Retrieved July 14, 2009 from http://www.statcan.gc.ca/pub/71-211-x/71-211-x2009 000-eng.pdf

[6] MacDonald, C.J., Stodel, E.J., Thompson, T.L., \& Casimiro, L., 2009. W(e)Learn: A Framework for Interprofessional Education. International Journal of Electronic Healthcare, 5(1), pp. 33-47. doi:10.1504/IJEH.2009.026271

[7] Creswell, J. W., \& Plano Clark, V. L., Designing and Conducting Mixed Methods Research $2^{\text {nd }}$ ed. Thousand Oaks, CA: Sage, 2010.

\section{AUTHORS}

C. J. MacDonald, EdD is a full professor at the Faculty of Education, University of Ottawa (e-mail: cjmacdon@uottawa.ca)

H. Kellam, MA is a PhD student in the Faculty of Education at the University of Ottawa (email: hkell094@uottawa.ca)

R. Sun, MEd is a PhD candidate in the Faculty of Education at the University of Ottawa (e-mail: rsun028@uottawa.ca )

D. Archibald, $\mathbf{P h D}$ is an educational researcher in the Department of Family Medicine at the University of Ottawa (e-mail: darchibald@bruyere.org)

E. Stodel, PhD is Founder and Principal of Learning 4 Excellence (e-mail: estodel@learning4excellence.com )

D. Puddester, MD, MEd is an associate professor in the Faculty of Medicine, University of Ottawa and Director of the Faculty Wellness Program (e-mail: wellness@uottawa.ca)

This work was supported in part by the Ontario Ministry of Health and Long-Term Care, the University of Ottawa, the Canadian Medical Foundation, and the Canadian Medical Association.

This work has been endorsed by the Canadian Association of Interns and Residents and the Fédération des médecins résidents du Québec

Submitted, April $14^{\text {th }}, 2011$. Published as resubmitted by the authors May $2^{\text {nd }}, 2011$. 